\title{
UCRL-JRNL-224385
}

LAW RENCE LIVERMORE N A T IO N A L LABORATORY

Experimental Tests Of

Paleoclassical Transport

J. D. Callen, J. K. Anderson, T. C. Arlen, G. Bateman, R. V. Budny, T. Fujita, C. M. Greenfield, M. Greenwald, R. J. Groebner, D. N. Hill, G. M. D. Hogeweij, S. M. Kaye, A. H. Kritz, E. A. Lazarus, A. C. Leonard, M. A. Mahdavi, H. S. McLean, T. H. Osborne, A. Y. Pankin, C. C. Petty, J. S. Sarff, H. E. St. John, W. M. Stacey, D. Stutman, E. J. Synakowski, K. Tritz

September 13, 2006

Nuclear Fusion 
This document was prepared as an account of work sponsored by an agency of the United States government. Neither the United States government nor Lawrence Livermore National Security, LLC, nor any of their employees makes any warranty, expressed or implied, or assumes any legal liability or responsibility for the accuracy, completeness, or usefulness of any information, apparatus, product, or process disclosed, or represents that its use would not infringe privately owned rights. Reference herein to any specific commercial product, process, or service by trade name, trademark, manufacturer, or otherwise does not necessarily constitute or imply its endorsement, recommendation, or favoring by the United States government or Lawrence Livermore National Security, LLC. The views and opinions of authors expressed herein do not necessarily state or reflect those of the United States government or Lawrence Livermore National Security, LLC, and shall not be used for advertising or product endorsement purposes. 


\section{Experimental Tests Of Paleoclassical Transport}

J.D. Callen ${ }^{1}$, J.K. Anderson ${ }^{1}$, T.C. Arlen ${ }^{2,3}$, G. Bateman ${ }^{4}$, R.V. Budny ${ }^{5}$, T. Fujita ${ }^{6}$, C.M. Greenfield ${ }^{3}$, M. Greenwald ${ }^{7}$, R.J. Groebner ${ }^{3}$, D.N. Hill ${ }^{3}$, G.M.D. Hogeweij ${ }^{8}$, S.M. Kaye ${ }^{5}$, A.H. Kritz ${ }^{4}$, E.A. Lazarus ${ }^{9}$, A.C. Leonard ${ }^{3}$, M.A. Mahdavi ${ }^{3}$, H.S. McLean ${ }^{10}$, T.H. Osborne ${ }^{3}$, A.Y. Pankin ${ }^{4}$, C.C. Petty ${ }^{3}$, J.S. Sarff ${ }^{1}$, H.E. St. John ${ }^{3}$, W.M. Stacey ${ }^{11}$, D. Stutman ${ }^{12}$, E.J. Synakowski ${ }^{10}$, K. Tritz ${ }^{12}$

${ }^{1}$ University of Wisconsin, Madison, WI 53706-1609 USA

${ }^{2}$ California Polytechnic State University, San Luis Obispo, CA 93407 USA

${ }^{3}$ General Atomics, San Diego, CA 92186-5608 USA

${ }^{4}$ Lehigh University, Bethleham, PA 18015-3182 USA

${ }^{5}$ Princeton Plasma Physics Laboratory, Princeton, NJ 08543-0451 USA

${ }^{6}$ Naka Site, JAEA, 801-1 Mukouyama, Naka, Ibaraki, 311-0193, JAPAN

${ }^{7}$ Massachusetts Institute of Technology, Cambridge, MA 02139 USA

${ }^{8}$ FOM Institute for Plasma Physics Rijnhuizen, 3430 BE Nieuwegein, NETHERLANDS

${ }^{9}$ Oak Ridge National Laboratory, Oak Ridge, TN 37831 USA

${ }^{10}$ Lawrence Livermore National Laboratory, Livermore, CA 94551-0808 USA

${ }^{11}$ Georgia Tech, Atlanta, GA 30332 USA

${ }^{12}$ Johns Hopkins University, Baltimore, MD 21218 USA

e-mail of main author: callen@engr.wisc.edu

Abstract. Predictions of the recently developed paleoclassical transport model are compared with data from many toroidal plasma experiments: electron heat diffusivity in DIII-D, C-Mod and NSTX ohmic and near-ohmic plasmas; transport modeling of DIII-D ohmic-level discharges and of the RTP ECH "stair-step" experiments with eITBs at low order rational surfaces; investigation of a strong eITB in JT-60U; H-mode $T_{e}$ edge pedestal properties in DIII-D; and electron heat diffusivities in non-tokamak experiments (NSTX/ST, MST/RFP, SSPX/spheromak). The radial electron heat transport predicted by the paleoclassical model is found to agree with a wide variety of ohmic-level experimental results and to set the lower limit (within a factor $\sim 2$ ) for the radial electron heat transport in most resistive, current-carrying toroidal plasmas - unless it is exceeded by fluctuation-induced transport, which often occurs in the edge of L-mode plasmas and when the electron temperature is high ( $\gtrsim T_{e}^{\text {crit }} \simeq B^{2 / 3} \bar{a}^{1 / 2}$ $\mathrm{keV}$ ) because then paleoclassical transport becomes less than gyro-Bohm-level anomalous transport.

\section{Introduction}

A new model for an irreducible minimum level of radial electron heat transport, the paleoclassical model, was introduced at the 2004 IAEA meeting [1a]; its basic features [1b] and details [1c] are now published. The key hypothesis of the model is that in resistive, current-carrying toroidal plasmas electron guiding centers diffuse radially with thin annuli of poloidal magnetic flux on the magnetic ("skin") diffusion time scale. This key hypothesis was originally motivated phenomenologically [1c]; recently, a "first principles" derivation of it has been developed [2]. This paper carries the initially encouraging comparisons with experimental data [1a] to a higher level via a number of more detailed comparisons of paleoclassical electron heat transport with data from a variety of toroidal plasma experiments. It also seeks to determine the situations (mainly ohmic-level plasmas and in the cooler plasma edge) where paleoclassical radial electron heat transport is dominant. Most comparisons are with well-characterized, previously published experimental data. In general, "typical best case" comparisons are shown in the figures; the text comments on other comparisons and in particular cases where the paleoclassical model does not represent the data well. The main comparisons are between the radial electron heat diffusivities predicted by the paleoclassical model and those inferred from "power balance" analyses; since typical error bars in both the theory [1] and experimental data analysis are of order a factor of two, agreement within this margin will be considered satisfactory. Some dynamic modeling tests are also performed.

\section{Brief Summary Of Paleoclassical Model}

The paleoclassical radial electron heat transport to be added to the right of an electron energy balance equation, and the implied radial electron heat diffusivity $\chi_{e}^{\mathrm{pc}}$ and magnetic field diffusivity $D_{\eta}$ are [1a,1c]

$$
-\left\langle\nabla \cdot \mathbf{Q}_{e}^{\mathrm{pc}}\right\rangle=\frac{M+1}{V^{\prime}} \frac{\partial^{2}}{\partial \rho^{2}}\left(V^{\prime} \frac{\eta_{\|}^{\mathrm{nc}}}{\mu_{0} \bar{a}^{2}} \frac{3}{2} n_{e} T_{e}\right), \quad \chi_{e}^{\mathrm{pc}} \equiv \frac{3}{2}(M+1) D_{\eta}, \quad D_{\eta} \equiv \frac{\eta_{\|}^{\mathrm{nc}}}{\mu_{0}} \sim \frac{\eta_{0}}{\mu_{0}} \equiv \frac{1400 Z_{\mathrm{eff}}}{T_{e}(\mathrm{eV})^{3 / 2}},
$$

in which $\eta_{\|}^{\text {nc }}$ is the neoclassical parallel resistivity, the unity in $M+1$ represents the axisymmetric contribution [1c], and the helical multiplier $M$ and average minor radius $\bar{a}$ are [1a,1c]

$$
M=\frac{\min \left\{\ell_{\max }, \lambda_{e}, \ell_{n^{\circ}}\right\}}{\pi \bar{R} q} \simeq \frac{1}{\pi \bar{R} q} \frac{1}{1 / \lambda_{e}+1 / \ell_{\max }}, \quad \frac{1}{\bar{a}^{2}} \equiv \frac{\left\langle|\nabla \rho|^{2} / R^{2}\right\rangle}{\left\langle R^{-2}\right\rangle} \simeq \frac{1}{a^{2}} \frac{1+\kappa^{2}}{2 \kappa^{2}} .
$$




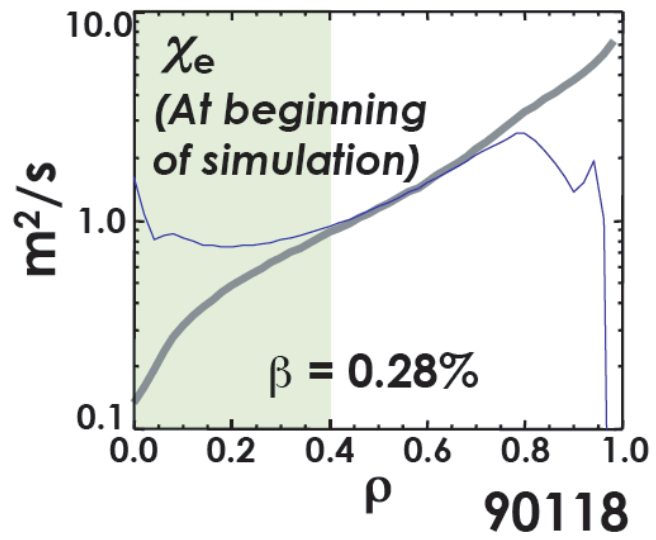

Figure 1: DIII-D electron heat diffusivity in ohmic-level beta-scan discharge: analysis (gray), paleo (blue), sawtooth (shaded).

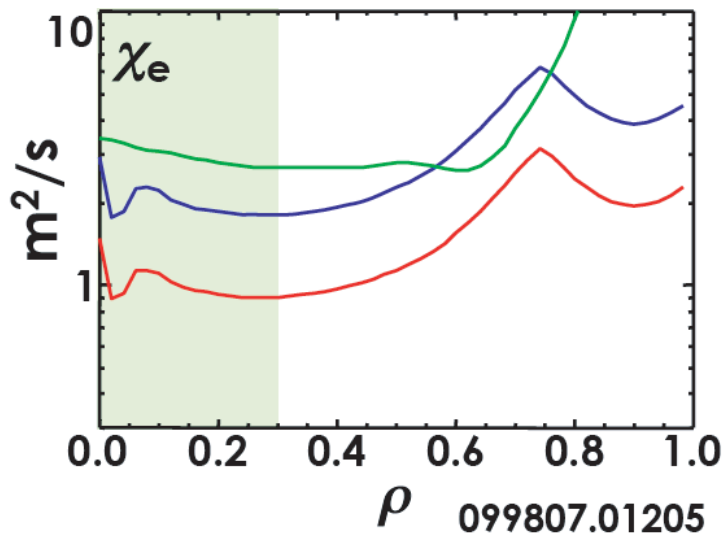

Figure 2: DIII-D electron heat diffusivity in Linear Ohmic Confinenment (LOC) regime: analysis (green), paleo (red), $2 \times$ paleo (blue).

The formulas after the $\simeq$ indicate the usually applicable smoothing formula for $M$ and an approximate formula for elliptical cross-section plasmas with $\kappa \equiv b / a \geq 1$. Further, $\lambda_{e} \simeq 1.2 \times 10^{16} T_{e}(\mathrm{eV})^{2} / n_{e} Z_{\text {eff }}$ is the electron collision length and $\ell_{\max }$ is the length over which magnetic field lines diffuse radially [1a,1c]:

$$
\ell_{\max }=\pi \bar{R} q n_{\max }, \quad n_{\max }=\left(\pi \bar{\delta}_{e}\left|q^{\prime}\right|\right)^{-1 / 2} ; \quad \max \left\{n_{\max }\right\}=\left(\pi^{2} \bar{\delta}_{e}^{2}\left|q^{\prime \prime}\right|\right)^{-1 / 3}, \quad \text { when }\left|q^{\prime}\right| \simeq 0 .
$$

Here, $\bar{\delta}_{e} \equiv c / \omega_{p} \bar{a}$ is the normalized em skin depth. Limits of the paleoclassical diffusivity are

$$
\underset{\lambda_{e}>\ell_{\max }}{\text { collisionless }} \chi_{e}^{\mathrm{pc}}=\frac{3}{2} \frac{\eta_{\|}^{\mathrm{nc}}}{\mu_{0}} n_{\max }, \quad \stackrel{\ell_{\max }>\lambda_{e}>\pi \bar{R} q}{\text { collisional }} \chi_{e}^{\mathrm{pc}}=\frac{3}{2} \frac{v_{T e}}{\pi \bar{R} q} \frac{c^{2}}{\omega_{p}^{2}} \frac{\eta_{\|}^{\mathrm{nc}}}{\eta_{0}}, \underset{\pi \bar{R} q>\lambda_{e}>\pi R}{\text { edge }} \chi_{e}^{\mathrm{pc}} \simeq \frac{10^{3} Z_{\mathrm{eff}}}{T_{e}(\mathrm{eV})^{3 / 2}} .
$$

Because $\chi_{e}^{\mathrm{pc}}$ scales with magnetic field diffusivity $D_{\eta}=\eta_{\|}^{\mathrm{nc}} / \mu_{0}$, it scales as $\bar{a}^{1 / 2} T_{e}^{-3 / 2}$ in the collisionless regime and decreases as $T_{e}$ increases. In contrast, drift-wave-type instabilities (ITG, DTEM, ETG) induce micro-turbulence and anomalous heat transport, which scale with the gyro-Bohm coefficient [1c] $\chi_{e}^{\mathrm{gB}} \simeq f_{\#} 3.2 T_{e}(\mathrm{keV})^{3 / 2} A_{i}^{1 / 2} / \bar{a} B^{2}$, that increase as $T_{e}$ increases. While the coefficient $f_{\#}$ is in general not well quantified, ITG simulations often find $\chi_{e} / \chi_{i} \lesssim 1 / 3$ and experimental results from TCV [3] indicate $f_{\#} \lesssim 1 / 3$, for all $R / L_{T e}$. Using $f_{\#} \simeq 1 / 3$, we can anticipate [1a] that, roughly speaking, below some $T_{e}$,

$$
T_{e} \leq T_{e}^{\mathrm{crit}} \simeq B(\mathrm{~T})^{2 / 3} \bar{a}(\mathrm{~m})^{1 / 2} \mathrm{keV}, \quad \text { paleoclassical electron heat transport is dominant? }
$$

Thus, we explore transport comparisons mainly in lower $T_{e}$ ohmic-level and edge plasmas.

\section{DIII-D Confinement Region Electron Heat Transport Comparisons}

Comparisons of paleoclassical predictions with $\chi_{e}^{\mathrm{pb}} \equiv\left\langle\mathbf{Q}_{e} \cdot \boldsymbol{\nabla} V\right\rangle /\left(-n_{e} \boldsymbol{\nabla} T_{e} \cdot \boldsymbol{\nabla} V\right)$ experimental "power balance" analysis data are most appropriate in the confinement region of tokamak plasmas, $0.4 \lesssim \rho \lesssim 0.9$ - because sawteeth often occur for $\rho \lesssim 0.4$ and transport data typically have large uncertainties for $\rho \gtrsim 0.9$. In the confinement region, tokamak plasmas are usually in the "collisionless" paleoclassical regime [1] where $\ell_{\max }$ dominates in (2) and $M=n_{\max } \sim 10$. Comparisons of $\chi_{e}^{\mathrm{pc}}$ with experimental $\chi_{e}^{\mathrm{pb}}$ data from 6 of the base ohmic-level $\left[T_{e}(0.4) \lesssim T_{e}^{\text {crit }} \sim 1-1.35 \mathrm{keV}\right]$ discharges in DIII-D beta [4a] and collisionality $[4 \mathrm{~b}]$ scans show reasonable agreement [5] - similar profiles and plasma parameter scaling, and usually within a factor of about 2 in magnitude [but low by a factor $\sim 3$ for low collisionality where $\left.T_{e}(0.4) \gtrsim T_{e}^{\text {crit }}\right]$, except near the edge. A "typical best case" comparison is shown in Fig. 1 . Here, $\chi_{e}^{\mathrm{pc}}$ decreases toward the edge $(\rho \gtrsim 0.8$ in Fig. 1$)$ because the collision length $\lambda_{e}$ becomes less than $\ell_{\max }$ and one transitions to the "collisional" (Alcator scaling) paleoclassical regime where $M=\lambda_{e} / \pi \bar{R} q$ and $\chi_{e}^{\mathrm{pc}} \propto T_{e}^{1 / 2} / n_{e} q$. The increase of $\chi_{e}^{\mathrm{pb}}$ with $\rho$ there could be caused by anomalous plasma transport induced by resistive ballooning modes (RBMs) [6] in this $T_{e} \lesssim 300 \mathrm{eV}$ region of these ohmic L-mode plasmas.

Figure 2 shows a similar comparison for a DIII-D plasma in the Linear Ohmic Confinement (LOC) regime [7] where $\tau_{E} \sim n_{e}$ and one would expect [1] to be in the collisional (Alcator-scaling) regime; while the agreement is reasonable over the critical region (for overall energy confinement) of $0.5 \lesssim \rho \lesssim 0.8$, this 


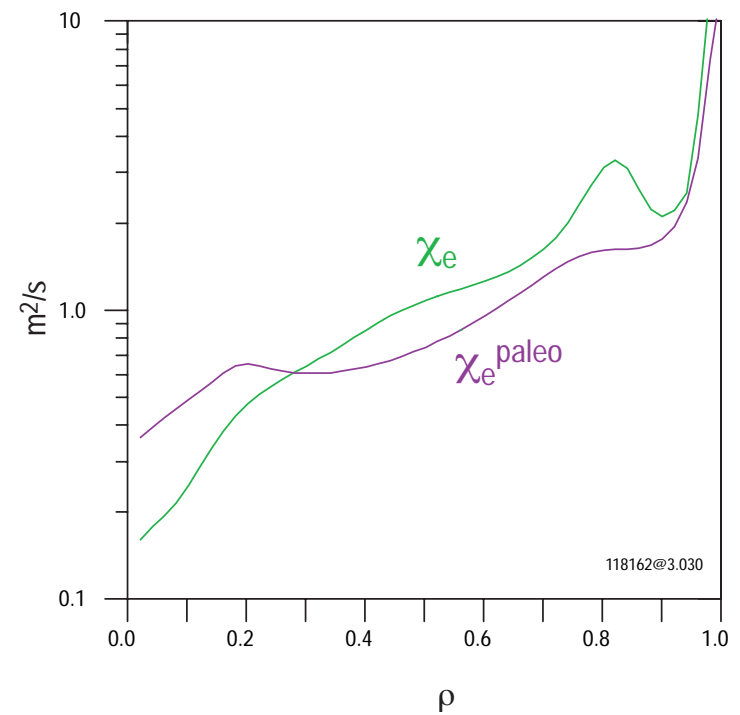

Figure 3: Profile of $\chi_{e}$ just before a sawtooth crash in DIII-D bean-shaped plasma [9].

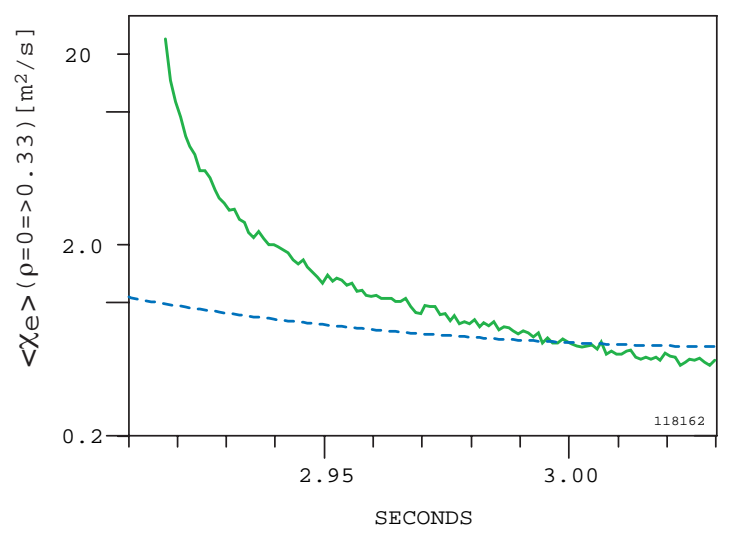

Figure 4: Average $\chi_{e}$ decays between sawtooth crashes: analysis (green), paleo (blue dashed) [9].

plasma is only marginally in the paleoclassical collisional regime there. A comparison in a higher density Saturated Ohmic Confinement (SOC) discharge, in which ITG turbulence was inferred to be present [7], found $\chi_{e}^{\mathrm{pc}}$ to be in the right range, but with the wrong (collisional) profile over this same radial region.

Dynamic ONETWO modeling of all these DIII-D discharges (from $\rho=0.9$ inward) using the paleoclassical transport model yields $T_{e}$ profiles in reasonable agreement (within $\lesssim 20 \%$ ) where $T_{e} \lesssim T_{e}^{\text {crit }}$. However, "thermal run away" occurs in simulations without a sawtooth modeling the central, sawtoothing region $\rho \lesssim 0.4$ because the collisionless $\chi_{e}^{\mathrm{pc}}$ decreases with increasing $T_{e}$.

Comparisons with DIII-D "hybrid" discharges [8] at $\rho \sim 0.5$ where $T_{e} \gtrsim 2.5 \mathrm{keV}\left(>T_{e}^{\mathrm{crit}} \simeq 1.3 \mathrm{keV}\right)$ show [5] that $\chi_{e}^{\mathrm{pc}}$ is a factor of 5-7 too small and has a different profile from $\chi_{e}^{\mathrm{pb}}$ for these discharges, which have micro-turbulence fluctuations (presumably due to ITG modes) and 3/2 NTMs in them. Thus, we conclude that for DIII-D ohmic-level plasmas the paleoclassical model predicts the $\chi_{e}$ magnitude and profile ( $\lesssim$ factor of 2 ) and $T_{e}$ profile within the confinement region - as long as $T_{e} \lesssim T_{e}^{\text {crit }}$ there.

There are, however, situations in DIII-D where $\chi_{e}^{\mathrm{pc}}$ sets the minimum level of transport even when $T_{e}>>T_{e}^{\text {crit }}$. Figure 3 shows such a case; it was obtained with a bean-shaped cross-section DIII-D plasma developed for sawtooth studies [9]. At the time shown (just before a sawtooth crash) it has $T_{e}(0) \simeq 2.5$ $\mathrm{keV}>>T_{e}^{\text {crit }} \simeq 1.3 \mathrm{keV}$. Also, Fig. 4 shows the core-averaged $\chi_{e}$ decays down to the paleoclassical level just before the next sawtooth crash. In a corresponding oval-shaped DIII-D plasma the $\chi_{e}^{\mathrm{pb}}$ values were a factor of 4-10 higher. The cause of the different transport properties are not presently understood [9].

\section{C-Mod Electron Heat Diffusivity, Critical $\mathbf{T}_{\mathrm{e}}$ Gradient And Power Flow}

Alcator C-Mod operates at higher magnetic field and thus has a higher $T_{e}^{\text {crit }}$ - about $1.6 \mathrm{keV}$ for $B \simeq 5.3 \mathrm{~T}$ and $\bar{a} \simeq 0.27 \mathrm{~m}$. A comparison of the $\chi_{e}^{\mathrm{pc}}$ with the experimental $\chi_{\mathrm{eff}}$, which includes both electron and ion heat diffusivities, for a well-diagnosed H-mode discharge [10] is shown in Fig. 5. For this discharge sawteeth influence $\rho \lesssim \rho_{\text {inv }} \simeq 0.35$ and $T_{e} \lesssim T_{e}^{\text {crit }} \simeq 1.6 \mathrm{keV}$ for $\rho>0.45$. For this case $\chi_{e}^{\mathrm{pc}}$ agrees well with experimental data in all three regimes in (4): collisionless for $\rho \lesssim 0.45$, collisional for $0.45 \lesssim \rho \lesssim 0.85$ and edge for $\rho \gtrsim 0.85$. Similar agreement is also obtained for an L-mode discharge [10].

The original paleoclassical papers [1] noted that the paleoclassical electron heat transport operator in (1) naturally includes a heat pinch or minimum temperature gradient effect. Specific forms of them were given $[1 \mathrm{a}, 1 \mathrm{c}]$ under the assumption that $M+1$ varies little with $\rho$. However, $M$ varies significantly for the

C-Mod data in Fig. 5 - from $\sim 20$ for $\rho \lesssim 0.45$ down to $<1$ for $\rho \gtrsim 0.85$. Thus, attempts to compare the critical $T_{e}$ gadient scale length in (58) of [1a] with the data in Fig. 5 failed, except for $\rho>0.85$ where 


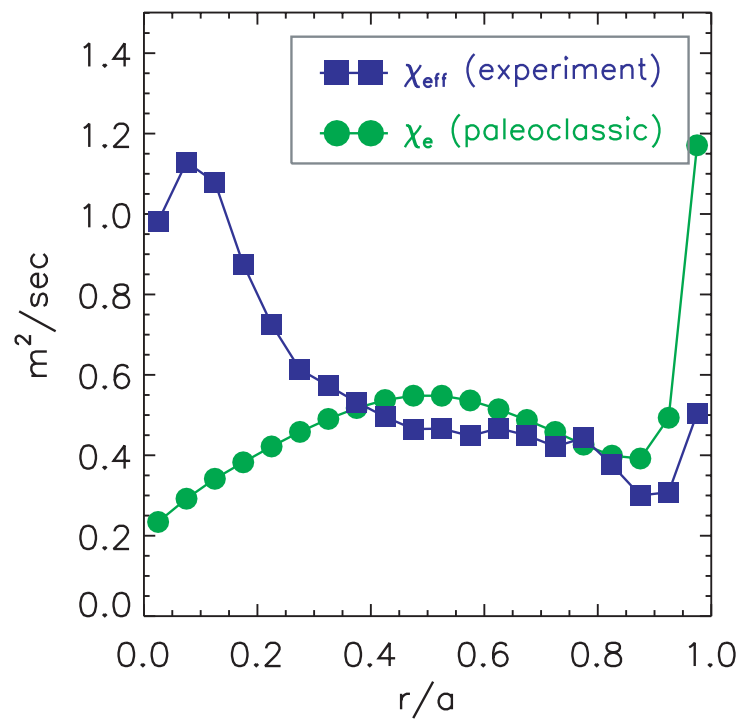

Figure 5: Electron heat diffusivity profile for CMod H-mode shot 960116027 [10].

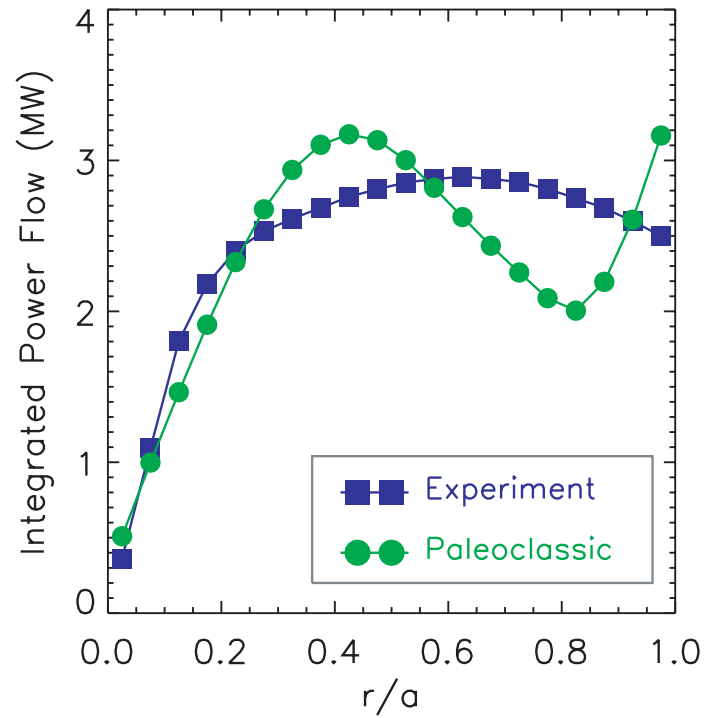

Figure 6: Electron power flow versus radius for C-Mod H-mode shot 960116027 [10].

it should be valid (because $M+1 \sim 1$ there) and did represent the data. As a check on the form of the paleoclassical transport operator, Fig. 6 shows that the volume integral of the first form in (1) agrees reasonably well with the experimental electron power flow for the H-mode discharge [10] in Fig. 5.

\section{Electron Internal Transport Barriers (eITBs) in RTP and JT-60U}

Near a low order rational surface (e.g., $q^{\circ} \equiv m^{\circ} / n^{\circ}=2 / 1$ ), $\ell_{n^{\circ}} \equiv \pi \bar{R} q^{\circ} n^{\circ}$ dominates in (2) and $M \simeq n^{\circ}$, which yields [1a,1c] electron "internal transport barriers" where $\chi_{e}^{\mathrm{pc}}$ is smaller by $\left(n^{\circ}+1\right) / n_{\max } \sim$ $0.2-0.5$ over widths determined by magnetic shear [1], as shown in Fig. 7 . These features produce transport barriers like those inferred [11] from the RTP "stair-step" experiments in which the central $T_{e}$ decreased abruptly as radially highly localized ECH was moved radially outward (in steps $\lesssim 0.01 a$ ) past

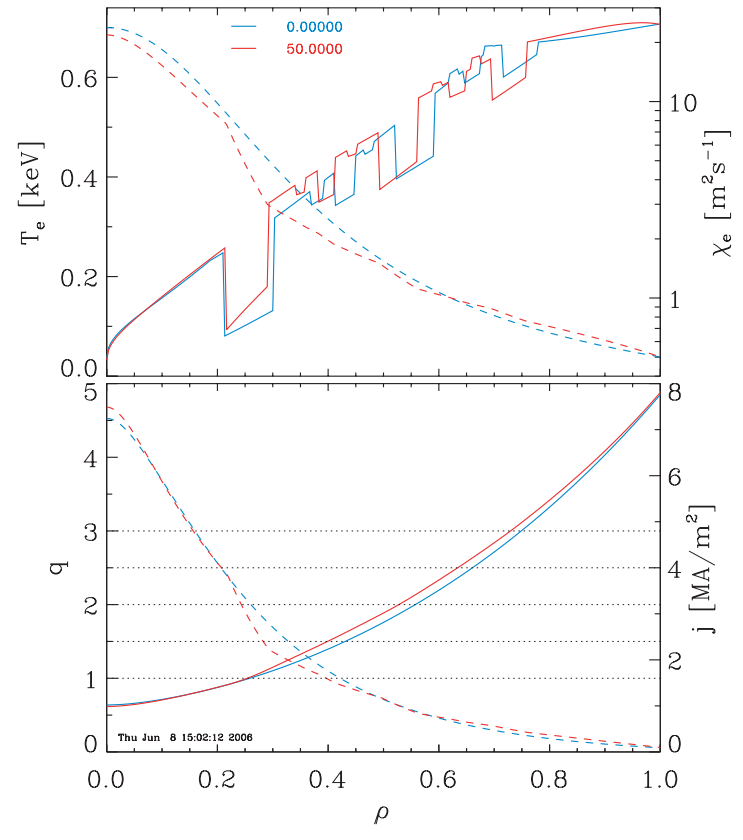

Figure 7: Modeling profiles for RTP ohmic discharge: initially (blue), after $50 \mathrm{~ms}$ (red). Largest eITBs are at $q=1 / 1,2 / 1,3 / 1[12]$.

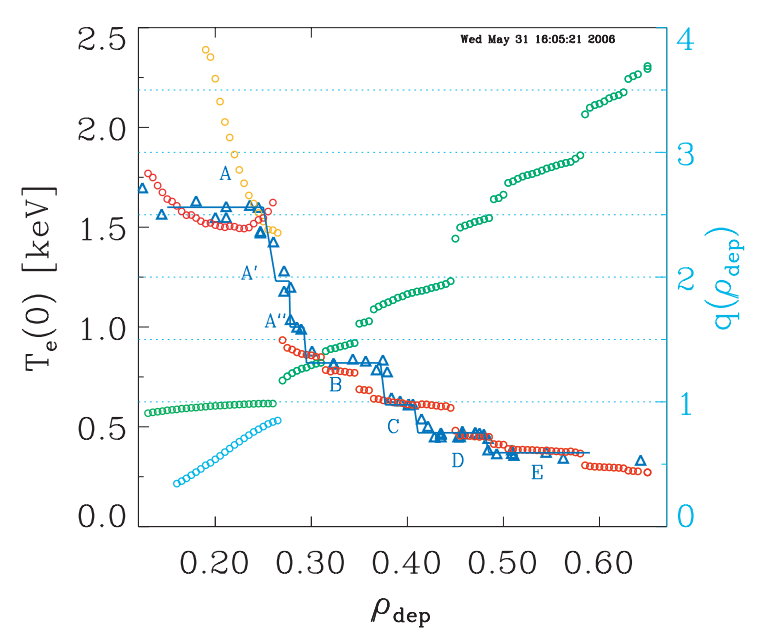

Figure 8: On axis $T_{e}$ as ECH deposition is moved radially outward: RTP experiment (blue), paleo modeling (red, orange) [12]. 


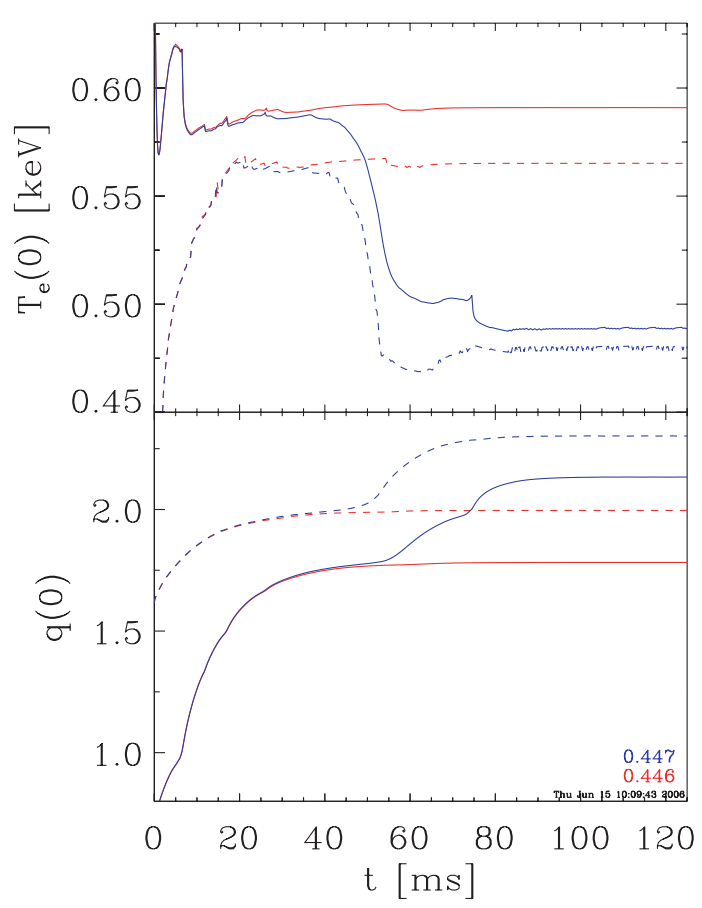

Figure 9: Evolution of central $T_{e}, q$ for $\mathrm{ECH}$ $\rho_{\text {dep }}=0.446$ (red), 0.447 (blue): RTP experiment (solid) and paleo modeling (dashed) [12].

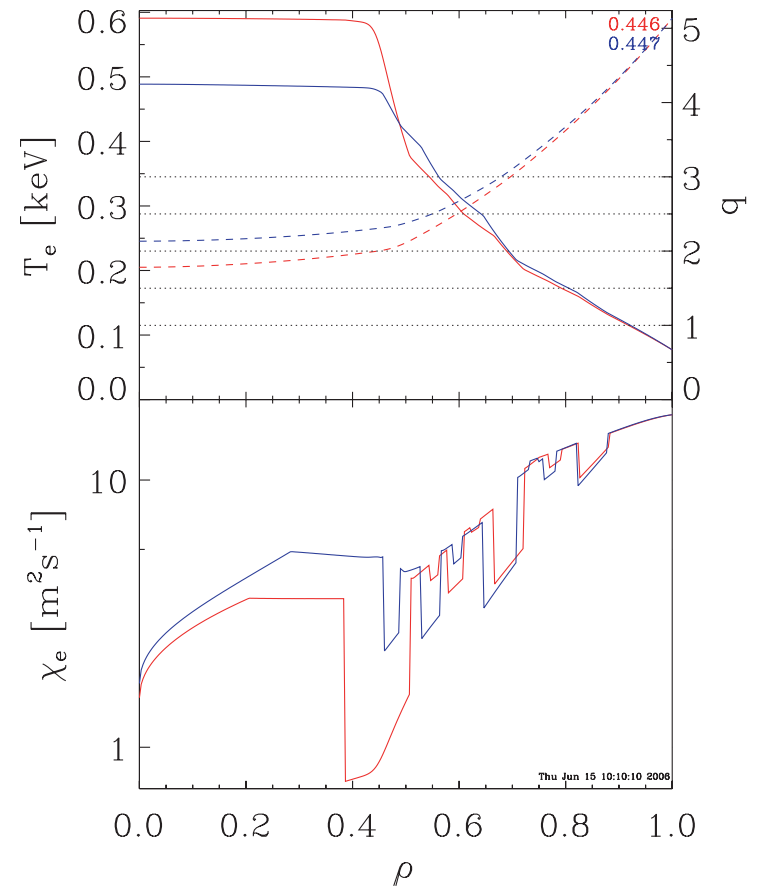

Figure 10: Corresponding paleoclassical modeling profiles of $T_{e}, q$ and $\chi_{e}$ for ECH $\rho_{\text {dep }}=0.446$ (red), 0.447 (blue) in RTP [12].

low order rational surfaces. Modeling of such RTP discharges with twice $\chi_{e}^{\mathrm{pc}}[12]$ is shown in Fig. 8 . [With $1 \times \chi_{e}^{\mathrm{pc}}$ only slightly higher $T_{e}(0)$ values and modified $q$ profiles are obtained.] For most of these cases $T_{e} \lesssim T_{e}^{\text {crit }} \simeq 0.7 \mathrm{keV}$ over most of the plasma and the collisionless $\chi_{e}^{\mathrm{pc}}$ is applicable for $\rho \lesssim 0.8$. The paleoclassical model results shown in Fig. 8 approximate the "stair step" details of the $T_{e}$ profile reasonably well. (However, the paleoclassical model does not reproduce the slightly hollow $T_{e}$ profiles that are observed experimentally for far off axis ECH which modify the barrier locations a bit [12].) As in the DIII-D dynamic modeling, "thermal runaway" occurs for $\rho \lesssim 0.25$ (orange points in Fig. 8) — unless a sawtooth $T_{e}$ relaxation model is used there (red points). The presence of eITBs at low order rational surfaces requires plasmas to come into a steady equilibrium [11] - apparently on the slow magnetic diffusion time scale. Paleoclassical modeling [12] of the evolution of two RTP plasmas with very closely spaced ECH deposition radii is shown in Fig. 9. The corresponding $T_{e}, q$ and $\chi_{e}^{\mathrm{pc}}$ profiles are shown in Fig. 10. The position sensitivity, temporal behavior and sharp transport bifurcations are well represented by the modeling of these cases in which the magnetic field diffusion time is $\tau_{\eta} \equiv a^{2} / 6 D_{\eta}(\rho=0) \sim 20 \mathrm{~ms}$.

Similarly, the original paleoclassical papers [1] proposed that the strong eITBs produced in JT-60U [13] were induced by an off-axis minimum in $q$ being at a low order rational surface causing a small $\chi_{e}^{\text {pc }} \sim n^{\circ} D_{\eta}$ there. While such an effect may help initiate an eITB, it is not relevant in fully developed JT-60U eITBs. Rather, the strong reversed shear inside $q_{\text {min }}$ decreases the collisionless $\chi_{e}^{\mathrm{pc}} \sim\left(q^{\prime}\right)^{-1 / 2}$ there. Then, if the anomalous transport due to micro-turbulence is negligible, $\chi_{e}^{\mathrm{pc}}$ can produce the low, irreducible minimum level of electron heat transport. An example of this for a strong eITB in JT-60U, for which $T_{e}^{\text {crit }} \simeq 2.4 \mathrm{keV}$, is shown in Figs. 11 and 12. The TRANSP analysis (Fig. 12) shows that the eITB occurs primarily inside the $q_{\min }$ surface at $\rho \simeq 0.575$ and that the reduction in $\chi_{e}$ there is well represented by the paleoclassical model in this JT-60U discharge in which a "reduction in the size of the turbulent structures is observed ... during the evolution of the internal transport barrier" [14]. Strongly reversed magnetic shear can also be important in the core of NSTX plasmas - see 7. below.

\section{H-Mode Edge $T_{e}$ Pedestals in DIII-D}

Figures 1, 2, 3 and 5 show that as $\rho$ approaches the separatrix, $\chi_{e}^{\mathrm{pc}}$ is first in the collisional regime where $\chi_{e}^{\mathrm{pc}} \propto T_{e}^{1 / 2} / n_{e} q$ decreases with increasing $\rho$. Further out where $\lambda_{e}<\pi R q, M<1$ and $\chi_{e}^{\mathrm{pc}} \propto T_{e}^{-3 / 2}$ increases as $T_{e}$ decreases further. Edge pedestal $n_{e}$ and $T_{e}$ profiles are shown in Fig. 13 for a well-diagnosed DIII-D H-mode discharge with $36 \mathrm{~ms}$ between ELM crashes. Figure 14 shows a comparison of $\chi_{e}^{\mathrm{pc}}$ with results from an integrated transport analysis code [15] of an analogous DIII-D shot 92976 which had a 


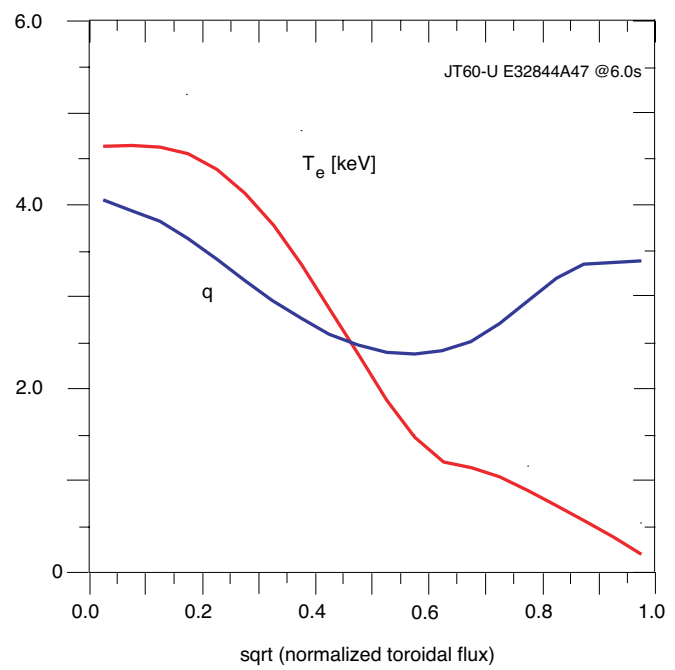

Figure 11: JT-60U $T_{e}, q$ profiles for a strong eITB, which is inside of $q_{\min }$ at $\rho \simeq 0.575$.

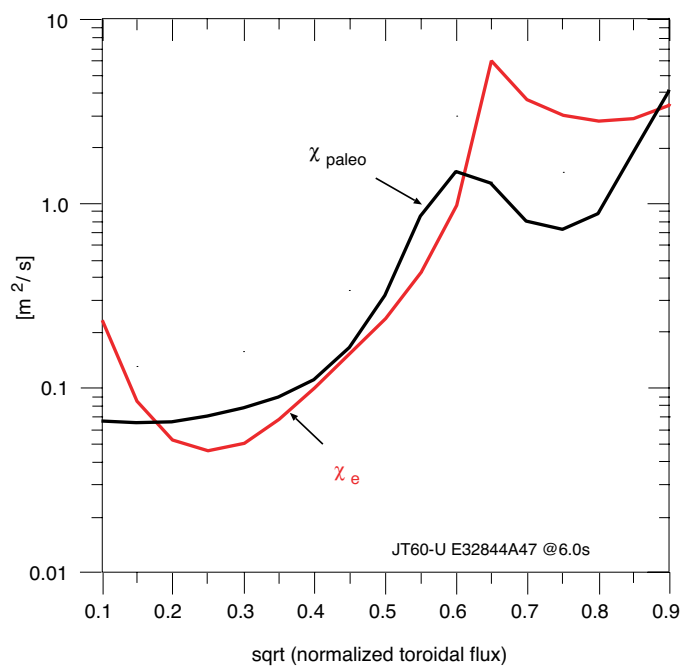

Figure 12: Comparison of TRANSP and paleoclassical $\chi_{e}$ for the JT-60U case in Fig. 11.

higher pedestal $n_{e}^{\text {ped }} \simeq 4.3 \times 10^{19} \mathrm{~m}^{-3}$ but lower $T_{e}^{\text {ped }} \simeq 300 \mathrm{eV}$. The paleoclassical $\chi_{e}$ compares favorably with the experimentally inferred $\chi_{e}$, especially in the near separatrix region $(\rho>0.96)$ where $\chi_{e}^{\mathrm{pc}} \propto T_{e}^{-3 / 2}$. The increasing $\chi_{e}^{\mathrm{pc}}$ in the near separatrix region causes the $T_{e}$ profile to have positive curvature (i.e., $\partial^{2} T_{e} / \partial \rho^{2}>0$ ), outside the $T_{e}$ "symmetry point" at $\rho=0.978$ in Fig. 13 . This aspect of the paleoclassical model is critical for producing appropriate modeling [16] of the edge $T_{e}$ pedestal, as illustrated in Fig. 15.

Specific paleoclassical model predictions have been developed for $T_{e}$ profile properties in an H-mode edge pedestal region [17]. Since in the near separatrix region $M<1$ and $n_{e} T_{e} D_{\eta} \propto n_{e} / T_{e}^{1 / 2}$, integrating the first equation in (1) from the separatrix inward the paleoclassical model predicts [17] $T_{e} \propto n_{e}^{2}$ or $\eta_{e} \equiv d \ln T_{e} / d \ln n_{e}=2$, in agreeement with ASDEX-U [18] and DIII-D data very close to the separatrix $\left(T_{e} \lesssim 200 \mathrm{eV}\right)$ [17]. This relation applies up to the point ( $\rho \simeq 0.95$ in Fig. 14) where $\lambda_{e} \lesssim \pi \bar{R} q / 2$ so that $M \lesssim 0.5$, beyond which $\chi_{e}^{\mathrm{pc}}$ stops decreasing so strongly and/or reaches a minimum causing a maximum $\left|\nabla T_{e}\right|$. Moving further inward from the separatrix, $\chi_{e}^{\mathrm{pc}}$ increases into the collisional regime. The pedestal $T_{e}$ is then determined by balancing paleoclassical transport against gyro-Bohm-scaled anomalous electron heat transport, which yields a prediction of $\beta_{e}^{\text {ped }} \equiv n_{e}^{\text {ped }} T_{e}^{\text {ped }} /\left(B^{2} / 2 \mu_{0}\right) \simeq\left(0.032 / f_{\#} A_{i}^{1 / 2}\right)\left(a / R_{0} q\right)\left(\eta_{\|}^{\text {nc }} / \eta_{0}\right)$ which is reasonably consistent with DIII-D pedestal data for $f_{\#} \sim 1$ [17] — see Fig. 16 .

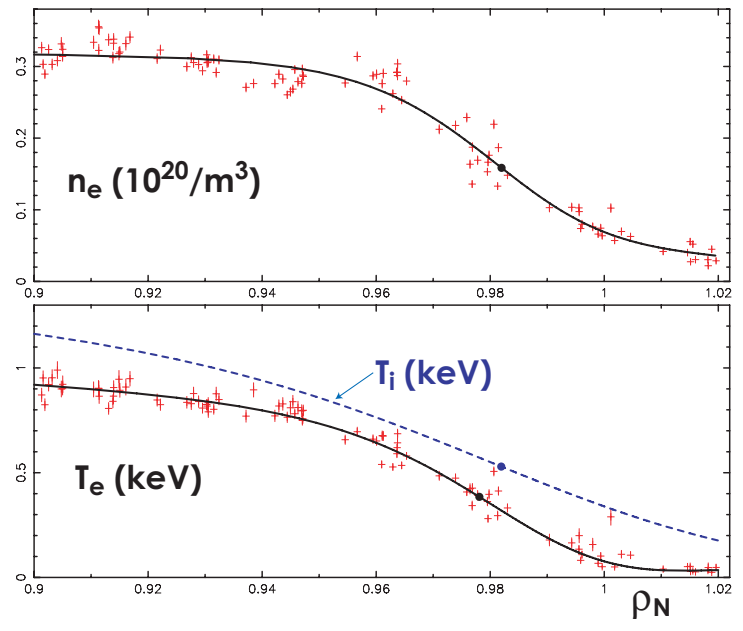

Figure 13: Edge pedestal $n_{e}$ and $T_{e}$ profiles for DIII-D shot 98889 , averaged over $80-90 \%$ of time to next ELM crash, around $4500 \mathrm{~ms}$.

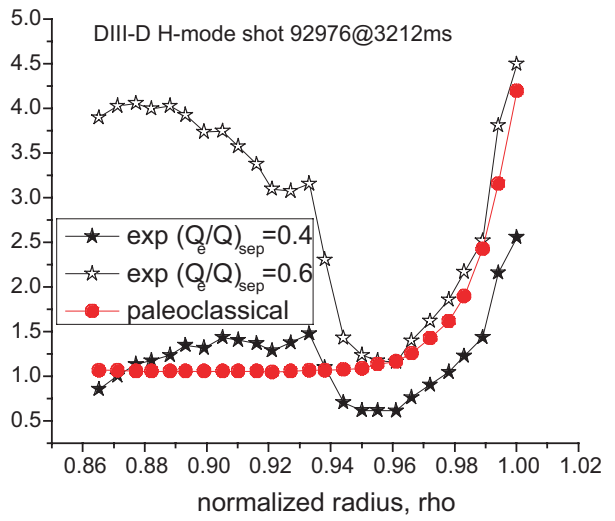

Figure 14: Transport analysis $\chi_{e}$ in DIII-D pedestal depends on electron fraction of power flowing through separatrix $\left(Q_{e} / Q\right)_{\text {sep }}[15]$. 


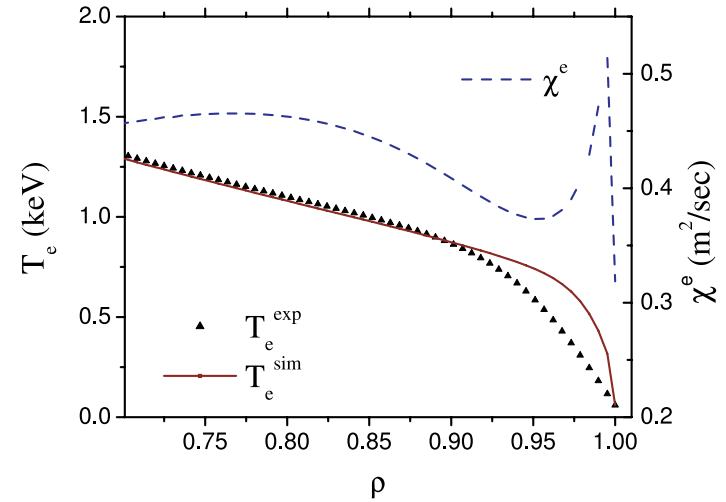

Figure 15: ASTRA modeling of the DIII-D discharge in Fig. 13 with paleoclassical model [16].

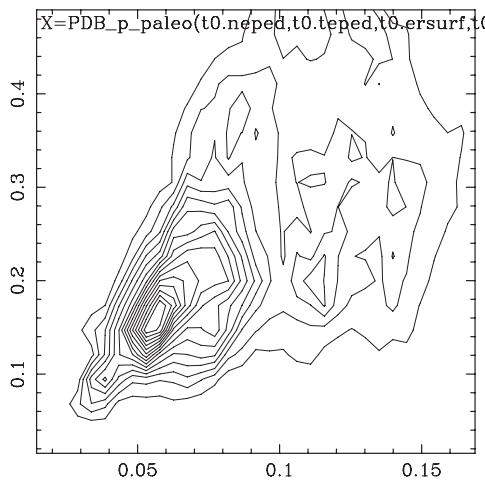

Figure 16: Database study of the paleoclassicalpredicted $\beta_{e}^{\text {ped }}$ versus $(a / R q)\left(\eta_{\|}^{\text {pc }} / \eta_{0}\right)$ in DIII-D.

\section{Non-tokamak Experiments: ST/NSTX, RFP/MST, Spheromak/SSPX}

The paleoclassical model [1a,1c] applies to axisymmetric toroidal current-carrying plasmas of all types - spherical tokamaks (STs), reversed field pinches (RFPs), and spheromaks - in regions where $\epsilon^{2}, B_{p}^{2} / B_{t}^{2}<<1$. Figure 17 shows that the paleoclassical model captures the decrease in core $\chi_{e}$ caused by moderately reversed shear $\left(q^{\prime}<0\right.$ for $\left.\rho<0.45\right)$ in an ohmic-level NSTX L-mode plasma, analogous to the $\rho<0.6 \mathrm{JT}-60 \mathrm{U}$ results in Fig. 12. The dotted line in Fig. 17 indicates the region where the zero shear, $\max \left\{n_{\max }\right\}$ formula at the end of (2) has been used. Figure 18 shows the ratio of the TRANSP analysis $\chi_{e}$ to the paleoclassical $\chi_{e}^{\mathrm{pc}}$ at $\rho=0.65$ for a variety L-mode NSTX discharges from the 2004 and 2005 campaigns. Two points about it are notable: 1) since all the data have ratios of about unity or greater the paleoclassical $\chi_{e}^{\mathrm{pc}}$ is setting the irreducible minimum electron thermal diffusivity; and 2) $\chi_{e}$ is at the paleoclassical level for $T_{e}$ less than about $0.65 B^{2 / 3} \mathrm{keV}$, but often above it for larger $T_{e}$. Similar comparisons for higher heating power NSTX H-modes [19] find that: the TRANSP $\chi_{e}$ usually significantly exceeds $\chi_{e}^{\mathrm{pc}}$ throughout the plasma, their minimum ratio is never below 0.5 , has a mean of about 4 and ranges up to 13 ; and all have $T_{e} / B^{2 / 3} \geq 0.5 \mathrm{keV}$. Since for these NSTX discharges $\kappa \simeq 1.9$ and $\bar{a} \simeq 0.8 \mathrm{~m}$, this implies that for these discharges $T_{e}^{\mathrm{crit}} \simeq 0.55-0.72 B^{2 / 3} \bar{a}^{1 / 2}$, which is less than a factor of two smaller than (5) or alternatively indicate $f_{\#} \sim 1-2$.

For quiescent RFP plasmas such as those in MST PPCD discharges [20], the magnetic fluctuations due to tearing modes are reduced; thus, the magnetic-flutter induced transport is reduced and the electron heat transport is reduced to tokamak levels. Figure 19 shows that the $\chi_{e}$ in these PPCD discharges is less than an order of magnitude above and has approximately the same shape as the paleoclassical $\chi_{e}^{\mathrm{pc}}$.

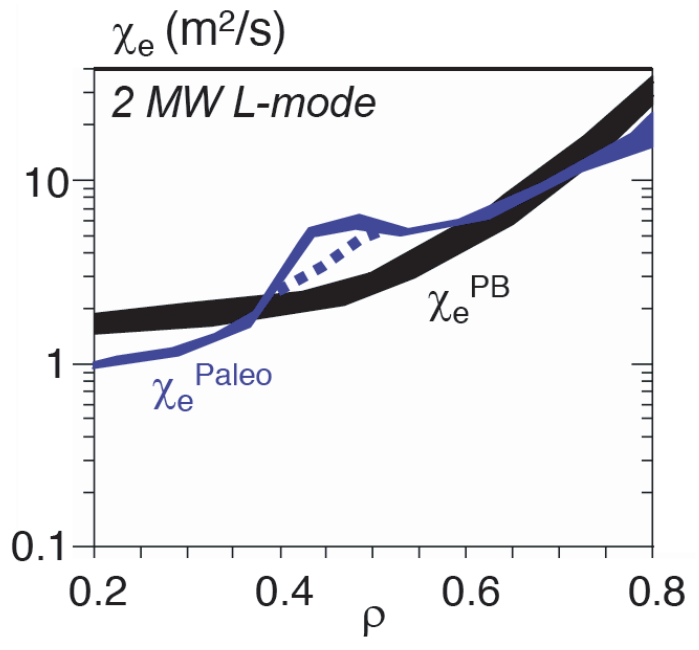

Figure 17: TRANSP and paleoclassical $\chi_{e}$ for an L-mode NSTX reversed shear plasma [19].

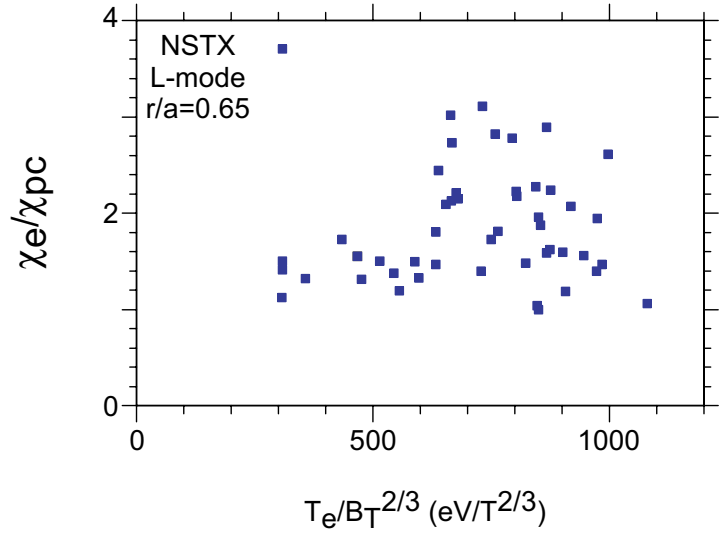

Figure 18: Ratio of TRANSP to paleoclassical $\chi_{e}$ vs. $T_{e}^{\text {crit }}$ parameter for NSTX L-modes. 


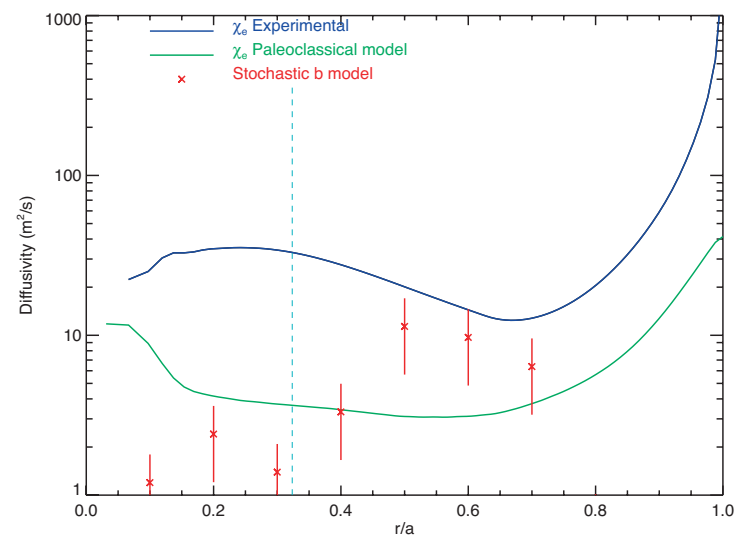

Figure 19: $\chi_{e}$ in quiescent (PPCD) MST plasmas [20]. Transport is not below paleoclassical.

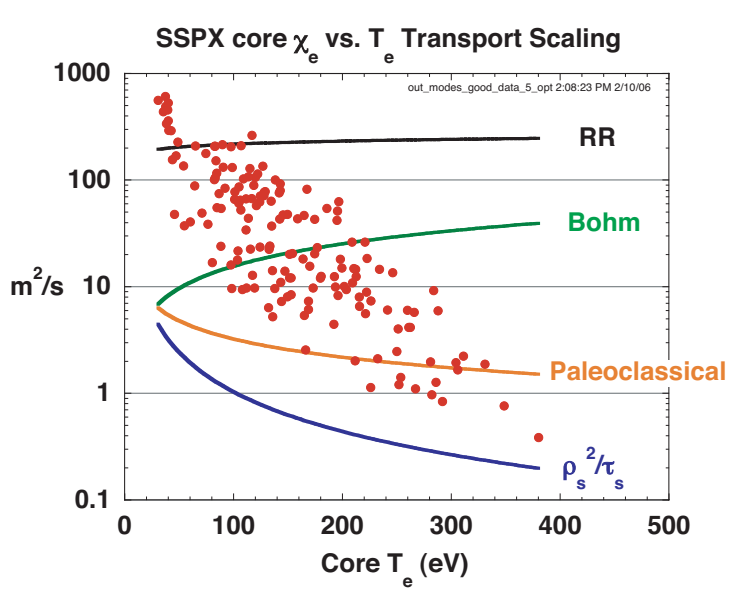

Figure 20: $\operatorname{SSPX} \chi_{e}$ on axis decreases as $T_{e}$ increases; paleoclassical may limit at high $T_{e}[21]$.

In the SSPX spheromak [21], as shown in Fig. 20, in $T_{e} \sim 100 \mathrm{eV}$ plasmas $n=1$ magnetic fluctuations are present and produce a magnetic-flutter level $\chi_{e}\left(\mathrm{RR}=\right.$ Rechester-Rosenbluth). As $T_{e}$ is increased (via magnetic flux increases), magnetic fluctuations and $\chi_{e}$ decrease. As indicated in Fig. 20, for $T_{e} \gtrsim 200$ $\mathrm{eV}$ the (collisional regime) paleoclassical $\chi_{e}^{\mathrm{pc}}$ may set the lower limit on electron heat transport.

\section{Conclusions About Paleoclassical Transport}

From these studies, we conclude that paleoclassical transport sets the irreducible minimum (factor $\sim 2$ ) electron heat transport in many resistive, current-carrying toroidal plasmas — when not exceeded by fluctuation-induced transport due to RBMs for $T_{e} \lesssim 300 \mathrm{eV}$ in L-mode plasmas, drift-type microturbulence (ITGs, TEMs, ETGs) for $T_{e} \gtrsim T_{e}^{\text {crit }} \equiv B^{2 / 3} \bar{a}^{1 / 2} \mathrm{keV}(\sim 0.7-2.4 \mathrm{keV}$ in present devices but $\sim 5 \mathrm{keV}$ in ITER) or magnetic fluctuations (Rechester-Rosenbluth $\left.\chi_{e}\right)$, or core $(\rho \lesssim 0.4)$ sawtooth effects.

This research was supported by the U.S. DoE grants and contracts DE-FG02-92ER54139 and DE-FC0205ER54184 (UW-Madison), DE-FC02-04ER54698 (GA), DE-FG02-92ER54141 (Lehigh), DE-AC02-76CH03073 (PPPL), DE-FG02-99ER54512 (MIT), DE-AC05-00OR22725 (ORNL), W-7405-ENG-48 (LLNL), DE-FG02-00ER54538 (GaTech) and DE-FG02-99ER54523 (JHU). The JAEA work was supported by the Japan Society for the Promotion of Science. The FOM work was supported by the EC European Fusion Programme and NWO.

[1] J.D. Callen, a) Nucl. Fus. 45, 1120 (2005); b) PRL 94, 055002 (2005); c) Phys. Plasmas 12, 092512 (2005).

[2] J.D. Callen, "Key hypothesis of paleoclassical model," UW-CPTC 06-XX, August 2006 (submitted to PoP).

[3] Y. Camenen, A. Pochelon et al., Plasma Phys. Control. Fusion 47, 1971 (2005) — see Figure 10.

[4] C.C. Petty, T.C. Luce et al., a) Nucl. Fusion 38, 1183 (1998); b) Phys. Plasmas 6, 909 (1999).

[5] T.C. Arlen et al., GP1 36, DPP-APS Denver (2005); C.M. Greenfield et al., TTF Myrtle Beach, SC (2006).

[6] B.A. Carreras, P.H. Diamond et al., PRL 50, 503 (1983); B.N. Rogers, J.F. Drake et al., PRL 81, 4396 (1998).

[7] C.L. Rettig, T.L. Rhodes et al., Phys. Plasmas 8, 2232 (2001); Plasma Phys. Control. Fusion 43, 1273 (2001).

[8] M.R. Wade, T.C. Luce, R.J. Jayakumar, P.A. Politzer, A.W. Hyatt et al., Nucl. Fusion 45, 407 (2005).

[9] E.A. Lazarus, F.L. Waelbroeck, T.C. Luce et al, Plasma Phys. Control. Fusion 48, L65 (2006).

[10] M. Greenwald, R.L. Boivin, F. Bombarda, P.T. Bonoli, C.L. Fiore et al., Nucl. Fusion 37, 793 (1997).

[11] a) N.J. Lopes Cardozo et al., PPCF 39, B303 (1997); b) G.M.D. Hogeweij et al., Nucl. Fus. 38, 1881 (1998).

[12] G.M.D. Hogeweij, J.D. Callen, H.J. de Blank, "Paleoclassical Electron Internal Transport Barriers in RTP," Controlled Fusion and Plasma Physics (Proc. 33th Eur. Conf., Roma, Italy, 2006), CD-ROM file P-4.148.

[13] a) T. Fujita et al., Phys. Rev. Lett. 78, 2377 (1997); b) Plasma Phys. Control. Fusion 46, A35 (2004).

[14] R. Nazikian, K. Shinohara, G.J. Kramer, E. Valeo et al., Phys. Rev. Lett. 94, 135002-1 (2005).

[15] W.M. Stacey and R.J. Groebner, Phys. Plasmas 13, 072510 (2005).

[16] A.Y. Pankin, G. Bateman, D.P. Brennan, D.D. Schnack, P.B. Snyder et al. Nucl. Fusion 46, 403 (2006).

[17] J.D. Callen, T.H. Osborne, W.M. Stacey, R.J. Groebner and M.A. Mahdavi, "Paleoclassical model for edge electron temperature pedestal," UW-CPTC 06-XX, September 2006 (currently being written).

[18] L.D. Horton, A.V. Chankin, Y.P. Chen et al., Nucl. Fusion 45, 856 (2005).

[19] D. Stutman, M.H. Redi, et al., "Studies of Improved Electron Confinement on NSTX" (to be published).

[20] J.S. Sarff, J.K. Anderson, T.M. Biewer, D.L. Brower et al., Plasma Phys. Control. Fusion 45 A457 (2003).

[21] H.S. McLean, R.D. Wood, B.I. Cohen, E.B. Hooper, D.N. Hill et al., Phys. Plasmas 13, 056105 (2006). 